\title{
Mixed Phase and Bound States in the Phase Diagram of the Extended Hubbard Model
}

\author{
M. BA̧K \\ Institute of Physics, A. Mickiewicz University \\ Umultowska 85, 61-614 Poznań, Poland
}

\begin{abstract}
The paper examines part of the ground state diagram of the extended Hubbard model, with the on-site attraction $U<0$ and intersite repulsion $W>0$ in the presence of charge density waves, superconducting and $\eta$-superconducting order parameters. We show the possibility of the stabilization of the mixed state, with all three nonzero order parameters, in the model with nearest neighbor interactions. The other result of the paper is application of the exact solution of the Schrödinger equation for the two-electron bound state, as an additional bound for the phase diagram of the model, resulting in the partial suppression of the superconducting state of the $s$-wave symmetry, in favor of the normal state phase.
\end{abstract}

PACS numbers: 71.10.Fd, 71.10.Hf, 71.27.+a

\section{Introduction}

The Hubbard model is known for its universality. The model and its extensions have been used in research of magnetism, superconductivity and especially high temperature superconductivity (HTS), other various phases in the solid, charge orderings, phase separation etc., in materials like high temperature superconductors, bismuthates, Chevrel phases, amorphous semiconductors, heavy fermion materials, systems with alternating valence to name the few (for a review see, e.g., Ref. [1]). Despite intensive research the results concerning the model come mostly from different approximations or numerical simulations. The exact results are scarce and concern mostly specific situation of one dimension (1D) or specific band fillings or specific limits: zero band width, infinite $U$, or infinite $d$. 
We can mention the exact solution in 1D obtained by the Bethe ansatz [2], Nagaoka ferromagnetism in repulsive, half-filled systems with one hole [3], flat band ferromagnetism [4], Lieb's ferrimagnetism [5], some bounds on correlation functions $[6-8]$ and a statement of necessity of bound states for existence of extended $s$-wave superconductivity in 2D systems with low electron density $n$, by Randeria et al. [9]. Let us also note that the mean-field BCS equations for superconductivity in the Hubbard model with effective attractive interaction between electrons, in the limit of vanishing electron density turn into the Schrödinger equations, which can also be solved exactly [10].

An interesting, unresolved question concerning the Hubbard model is: what happens when we increase the strength of the interaction? What phases do we obtain? Or does the system prefer phase separation? That was the conclusion of the paper by Robaszkiewicz and Pawłowski [11]. By the broken symmetry Hartree-Fock approach they established that the attractive extended Hubbard model (with nearest neighbor - nn - density-density interactions of strength $W$ added) in two dimensions for arbitrary electron density and moderate values of $|W|$ is superconducting in the ground state but becomes phase separated with increasing $|W|$ (except from $n=1$, where the ground state is in charge density wave phase for any $W \geq 0$ ). In the case of $W<0$ they obtained the phase of electron droplets and for $W>0$ a phase separation between charge density waves (CDW) with $n=1$ and a singlet on-site superconductivity (SS) (extended $s$-wave was not considered). The free energy of this phase separated state (PS[CDW/SS]) turned out to be lower than the energy of the homogeneous mixed state $(\mathrm{M}(\mathrm{CDW}+\mathrm{SS}))$. Stabilization of $\mathrm{M}$ state required longer than $\mathrm{nn}$ repulsive intersite interactions $\left(W_{2}\right)$ or increasing $|U|$. For $U=-\infty$ PS and M states were degenerated even for $W_{2}=0$.

In this paper it will be shown that including $\eta$-pairing (superconducting pairing with singlet pairs with center-of-mass momentum $\pi$ ) [12-14] into the Hamiltonian stabilizes mixed phase, already with intersite interactions $W$ restricted to the nn (in disagreement with Ref. [11]). It turns out that the phase separation PS[CDW/SS] exists only below certain critical value of $W$, above which the $\mathrm{M}$ phase can be stabilized.

\section{Low density limit}

It can be shown that in the low density limit the BCS equations go over to the Schrödinger equation for the bound pair [10]. In the center-of-mass coordinate system the wave function of the two particle bound state can be expanded as the sum of states with the definite relative momenta. Using that expansion we can obtain a set of self-consistent equations for the pair wave function in a position space, in terms of lattice Green functions [1, 15]. For the hypercubic lattices these equations were solved (see, e.g., [1]) and it has been found out that in one and two 
dimensions pairs for $W=0$ bind for any negative $U$, while in three dimensions there is a critical value for $W$. In the cited reference the formula was given for the $W_{\text {cr }}$ as a function of $U$ :

$$
\frac{\left|W_{\mathrm{cr}}\right|}{2 t}=\left(1+\frac{2 D}{U}\right)^{-1}+(C-1)^{-1}
$$

where $C=(1 / N) \sum_{k}\left(1-\gamma_{k} / z\right)^{-1}$ is the Watson integral. In Ref. [1] this formula was given only for the case of $W<0$ but in fact it is valid for any $U$ and $W$. What is more, there exists analogous formula, applicable not only in the low density limit but for any electron density. It was derived from the Hamiltonian after the Hartree-Fock linearization so it is not exact, but goes over into the exact Eq. (1) for $n \rightarrow 0$. The formula is given by

$$
W_{\text {cr }}=\frac{8 t^{2}}{\mu(1-n)+8 t I-2 \mu^{2} / U}, \quad \text { where } \quad I=\int_{-1}^{\mu} x \rho(x) \mathrm{d} x
$$

or for the rectangular density of states (DOS) $[16]: W_{\mathrm{cr}}\left[1+(n-1)^{2}\left(1+\frac{16 t}{U}\right)\right]=-4 t$.

\section{Phase separation}

The energy of a phase separated state, $E_{\mathrm{PS}}$, is calculated according to the formula

$$
E_{\mathrm{PS}}=m E_{+}\left(n_{+}\right)+(1-m) E_{-}\left(n_{-}\right)
$$

where $E_{ \pm}\left(n_{ \pm}\right)$are the values of $\langle H\rangle / N$ of the two separating phases at the $n=n_{ \pm}$ corresponding to the lowest energy homogeneous solution for a given phase. $m$ and $(1-m)$ are fractions of the system occupied by the phases $E_{+}$and $E_{-}$, respectively. There is also a similar equation connecting charge densities in the two coexisting phases

$$
m n_{+}+(1-m) n_{-}=n .
$$

Very useful is also the Maxwell construction

$$
\frac{\partial E_{-}\left(n_{-}\right)}{\partial n_{-}}=\frac{E_{+}\left(n_{+}\right)-E_{-}\left(n_{-}\right)}{n_{+}-n_{-}},
$$

which is equivalent to the condition of minimization of $E_{\mathrm{PS}}$ respectively $n_{-}$with fixed $n_{+}$and $n$. Taking into account Eqs. (4) and (5), Eq. (3) can be rewritten as

$$
E_{\mathrm{PS}}=E_{+}\left(n_{+}\right)-\left(n_{+}-n\right) \frac{\partial E_{-}\left(n_{-}\right)}{\partial n_{-}} .
$$

There is also another, equivalent but more intuitive approach using the Gibbs energies $G=F-\mu n$. The independent variable for $G$ is chemical potential $\mu$ instead of charge density $n$ for $F$. Let us note that instead of the analogue of Eq. (4), with $\mu_{ \pm}$instead of $n_{ \pm}$, we have $\mu_{+}=\mu_{-}$, because this is the condition of equilibrium, or rather the definition of equilibrium! (Eq. (4) does not apply also because $\mu$ is not an extensive quantity). There is only one value of chemical 
potential, fixed and the same for both phases. This way the whole construction simplifies. The procedure is as follows: we find solutions for the homogeneous phases, then we plot their Gibbs energies vs. $\mu$ in one figure and we look for the intersections of $G$ 's belonging to the different phases. Such intersection means a coexistence of the two phases with the same $\mu$, i.e., in equilibrium. The same value of $\mu$ usually translates into different values of $n_{+}$and $n_{-}$in the two phases under consideration and that is nothing else but the (definition of) phase separation, which appears on the $F-n$ phase diagram for the electron density $n$, constructed according to Eq. (4).

Some remarks are due:

- let us note that the use of $G$ and chemical potential makes it clear that $n_{+}$ and $n_{-}$are independent of $n$, as there is usually one intersection point of the Gibbs energies. In other words, $m$ is a linear function of $n$ :

$$
m=\frac{1}{n_{+}-n_{-}} n-\frac{n_{-}}{n_{+}-n_{-}} ;
$$

- let us note that Eq. (3) gives boundaries for $E_{\mathrm{PS}}$. By the definition in Eq. (3), $E_{\mathrm{PS}}$ must be somewhere between $E_{+}\left(n_{+}\right)$and $E_{-}\left(n_{-}\right)$. By the same reasoning that led us to Eq. (7) we can say that the dependence of phase separated states on $n$ should be strictly linear, with the same coefficients as in Eq. (7). That is nothing else but the Maxwell condition restated;

- the same considerations as above apply to the electron density: phase separated state can exist only for charge densities $n_{-}<n<n_{+}$. This is an important bound, which can be easily overlooked, as there are formal solutions for free energy of phase separated state also outside that range;

- a simple implication of the preceding points, which we will call theorem 1: we cannot obtain a phase transition by changing $n$ between two phase separated states having "a state in common" for the same fixed density $n_{+}$, i.e. we cannot have phase transition between states like $\operatorname{PS1}[\mathrm{CDW}(n=1) /$ phase1] and $\operatorname{PS} 2\left[\mathrm{CDW}(n=1) /\right.$ phase2]. The lines depicting $F_{\mathrm{PS} 1}$ and $F_{\mathrm{PS} 2}$ will begin in the same point and will extend in different directions;

- and last but not the least is the notion that we have also bounds for homogeneous phases: the phases can exist only for the positive compressibility, i.e. in the range of $\mu$, for which $\partial \mu / \partial n>0$.

\section{The model}

We start from the extended Hubbard model

$$
H=\sum_{i j \sigma} t_{i j} c_{i \sigma}^{\dagger} c_{j \sigma}+U \sum_{i} n_{i \uparrow} n_{i \downarrow}+\frac{1}{2} W \sum_{i j} n_{i \sigma} n_{j \sigma^{\prime}}-\mu \sum_{i} n_{i}
$$

in standard notation. We use the Hartree-Fock approach. In the mean-field Hamiltonian we retain charge density order parameter $\Delta_{\mathrm{c}}$ and singlet superconductivity 
order parameters: $\Delta_{0}$ and $\Delta_{Q}$, for on-site and $\eta$-pairing respectively (see also Ref. [11]):

$$
\begin{aligned}
H_{0}= & \sum_{k \sigma}\left(\varepsilon_{k}-\bar{\mu}\right) c_{k \sigma}^{\dagger} c_{k \sigma}+\frac{1}{2} \sum_{k \sigma}\left(\Delta_{\mathrm{c}} c_{k \sigma}^{\dagger} c_{k+Q \sigma}+\text { h.c. }\right) \\
& +\sum_{k}\left(\Delta_{0} c_{k \uparrow}^{\dagger} c_{-k \downarrow}^{\dagger}+\text { h.c. }\right)+\sum_{k}\left(\Delta_{Q} c_{k+Q \uparrow}^{\dagger} c_{-k \downarrow}^{\dagger}+\text { h.c. }\right)+C,
\end{aligned}
$$

where the order parameters are given by

$$
\begin{aligned}
& \Delta_{\mathrm{c}}=\frac{1}{N} \sum_{k \sigma}\left(-z W+\frac{U}{2}\right)\left\langle c_{k+Q \sigma}^{\dagger} c_{k \sigma}\right\rangle, \\
& \Delta_{0}=\frac{U}{N} \sum_{k_{2}}\left\langle c_{-k_{2} \downarrow} c_{k_{2} \uparrow}\right\rangle, \\
& \Delta_{Q}=\frac{U}{N} \sum_{k_{2}}\left\langle c_{k_{2} \downarrow} c_{-k_{2}+Q \uparrow}\right\rangle,
\end{aligned}
$$

where $z$ is number of nearest neighbors, $\bar{\mu}=\mu-(U / 2+z W) n, W_{k}=W \gamma_{k}$, $\varepsilon_{k}=-t \gamma_{k}, \gamma_{k}=2 \sum_{\alpha}^{d} \cos k_{\alpha}, \alpha \in(x, y, z)$ and $d=z / 2$ is dimensionality of the hypercubic lattice; $\varepsilon_{k} \in\left\langle-\varepsilon_{0}, \varepsilon_{0}\right\rangle$ where $\varepsilon_{0} \equiv D=z t$. In the following all values of dimension energy will be given in units of $D$ and the simplest, zero temperature forms of the equations will be most often used. The constant $C$ takes the form

$$
C=\frac{1}{4}(U+2 W z) n^{2}-\frac{\left|\Delta_{c}\right|^{2}}{U-2 W z}-\frac{\left|\Delta_{0}\right|^{2}}{U}-\frac{\left|\Delta_{Q}\right|^{2}}{U} .
$$

After diagonalization we obtain Hamiltonian $H_{0}$ with two eigenenergies $A_{k}^{ \pm}$:

$$
\begin{aligned}
A_{k}^{ \pm} & = \\
& \sqrt{\varepsilon_{k}^{2}+\bar{\mu}^{2}+\Delta_{0}^{2}+\Delta_{Q}^{2}+\Delta_{\mathrm{c}}^{2} \pm 2 \sqrt{\varepsilon_{k}^{2}\left(\bar{\mu}^{2}+\Delta_{Q}^{2}\right)+\left(\bar{\mu} \Delta_{\mathrm{c}}-\Delta_{0} \Delta_{Q}\right)^{2}}} .
\end{aligned}
$$

In terms of the eigenstates of $H_{0}$ the free energy is given by the standard formula

$$
F_{0}=-\frac{1}{\beta} \ln \operatorname{Tr} \exp \left(-\beta H_{0}\right)+\mu N_{\mathrm{e}}
$$

which yields

$$
\frac{F_{0}}{N}=\bar{\mu}(n-1)+C-\frac{1}{\beta N} \sum_{k} \ln \left(4 \cosh \frac{\beta A_{k}^{+}}{2} \cosh \frac{\beta A_{k}^{-}}{2}\right) .
$$

The equations for the order parameters and chemical potential are obtained in a standard way, by minimization of the above expression. For the most general mixed, homogeneous phase with all the order parameters present the equations take the form 


$$
\begin{aligned}
& 2(n-1)=\bar{\mu}\left(I_{0}+J_{2}\right)+\Delta_{\mathrm{c}}\left(\bar{\mu} \Delta_{\mathrm{c}}-\Delta_{0} \Delta_{Q}\right) J_{0}, \\
& \frac{-4 \Delta_{Q}}{U}=\Delta_{Q}\left(I_{0}+J_{2}\right)-\Delta_{0}\left(\bar{\mu} \Delta_{\mathrm{c}}-\Delta_{0} \Delta_{Q}\right) J_{0}, \\
& \frac{-4 \Delta_{0}}{U}=\Delta_{0} I_{0}-\Delta_{Q}\left(\bar{\mu} \Delta_{\mathrm{c}}-\Delta_{0} \Delta_{Q}\right) J_{0}, \\
& \frac{-4 \Delta_{\mathrm{c}}}{U-2 W z}=\Delta_{\mathrm{c}} I_{0}+\bar{\mu}\left(\bar{\mu} \Delta_{\mathrm{c}}-\Delta_{0} \Delta_{Q}\right) J_{0},
\end{aligned}
$$

where

$$
\begin{aligned}
& I_{0}=\frac{1}{N} \sum_{k}\left(\frac{1}{A_{k}^{+}}+\frac{1}{A_{k}^{-}}\right), \\
& J_{n}=\frac{1}{N} \sum_{k}\left(\frac{1}{A_{k}^{+}}-\frac{1}{A_{k}^{-}}\right) \frac{\varepsilon_{k}^{n}}{\sqrt{\varepsilon_{k}^{2}\left(\bar{\mu}^{2}+\Delta_{Q}^{2}\right)+\left(\bar{\mu} \Delta_{\mathrm{c}}-\Delta_{0} \Delta_{Q}\right)^{2}}},
\end{aligned}
$$

where $A_{k}^{ \pm}$are given by Eq. (14). The equations for the mixed phases with smaller number of constituent phases (i.e., $\mathrm{M}(\mathrm{CDW}+\mathrm{SS}), \mathrm{M}(\mathrm{CDW}+\mathrm{SQ}), \mathrm{M}(\mathrm{SS}+\mathrm{SQ})$ ) as well as for pure phases can be obtained from Eqs. (17)-(22) by just dropping out respective order parameters. For the detailed form of the pure phase equations the reader is referred, e.g. to Refs. [11, 16].

\section{Results}

The results were obtained using rectangular DOS and $z=4$ as an approximation for two-dimensional square lattice. In Figs. 1a and b we plotted the results for the thermodynamical potentials. In part (a) we have shown all the solutions for the Gibbs energy (vs. chemical potential), without applying yet the bonds for $n \pm$ or checking the sign of $\partial \mu / \partial n$. All homogeneous phases are shown, except from $\eta$-pairing (denoted by $\mathrm{SQ}$ ) and mixed $\mathrm{M}(\mathrm{SS}+\mathrm{SQ}$ ) (SS denoting singlet, onsite superconductivity), which do not have solutions for $U>-2$. Nevertheless the phases: $\mathrm{M}(\mathrm{CDW}+\mathrm{SQ}), \mathrm{M}(\mathrm{CDW}+\mathrm{SS})$, upper branch of $\mathrm{M}(\mathrm{CDW}+\mathrm{SS}+\mathrm{SQ})$ (see lower inset) and upper branch of CDW for $n \neq 1$ must be excluded, because of the negative compressibility, connected with the downward curvature of these lines (compare also the upper inset - decreasing and increasing part of the plot corresponds to the opposite signs of compressibility and to the two branches of $\mathrm{M}(\mathrm{CDW}+\mathrm{SS}+\mathrm{SQ})$, from now on denoted as $\mathrm{M} 3$, in the main figure).

The remaining curves (and CDW one, for comparison) are a basis for plotting the free energy $F-n$ diagram, shown in part (b). The plot contains curves in the proper range of $n$, with all the bounds already applied. Apart from all the homogeneous phases fulfilling the condition $\partial \mu / \partial n>0$ (which corresponds to the downward curvature for $F(n)$ ) we can see also phase separated states indicated in (a) by the Gibbs energy plots crossings: PS[CDW/SS], PS[CDW/NO], PS[M3/SS] 

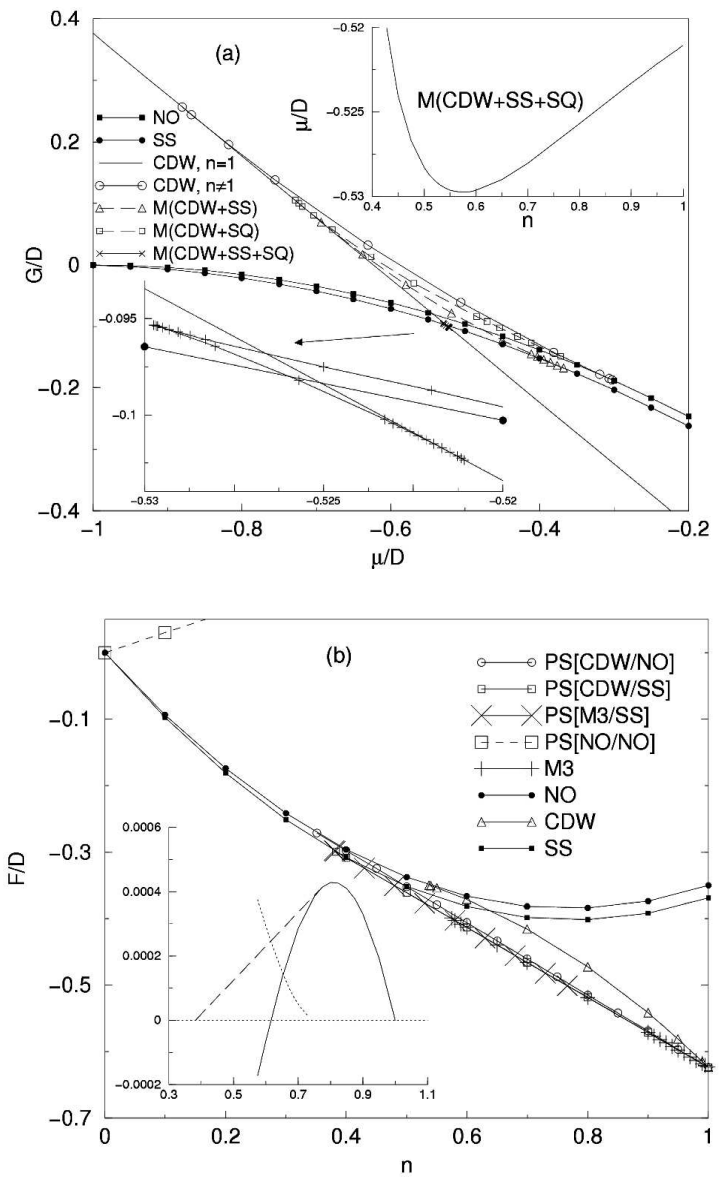

Fig. 1. (a) Gibbs energies $G$ vs. bare chemical potential $\mu$, for $U=-1$ and $W=0.2$. Lines are described in the figure. In the lower, left-hand corner in the inset, there is an enlargement of the area where the curves cross. In the inset in the upper right-hand corner there is plotted the dependence of chemical potential $\mu$ on charge density $n$ in M3 phase. (b) The free energy $F$ vs. electron density $n$ for $U=-1$ and $W=0.2$. Lines are described in the figure. In the inset there are the differences of free energies plotted — full line: $F(\mathrm{PS}[\mathrm{CDW} / \mathrm{SS}])-F(\mathrm{M} 3)$, dashed line: $F(\mathrm{PS}[\mathrm{CDW} / \mathrm{SS}])-F(\mathrm{PS}[\mathrm{M} 3 / \mathrm{SS}])$, dotted line: $F(\mathrm{M} 3)-F(\mathrm{PS}[\mathrm{M} 3 / \mathrm{SS}])$ for the range of $n$, where $\partial \mu / \partial n>0$ for each phase. Horizontal dotted line is the just a guide for an eye.

(and PS[NO/NO], of slightly different nature). The states phase separated with $\operatorname{CDW}(n=1)$ are connecting the point of energy $E^{\mathrm{CDW}}(n=1)$ with respective curves of the other state coming into separation, in a tangential way, in accordance with the Maxwell construction and our earlier remarks. Let us note that the CDW inside PS states is a phase with $n=1$ while CDW in mixed states is 

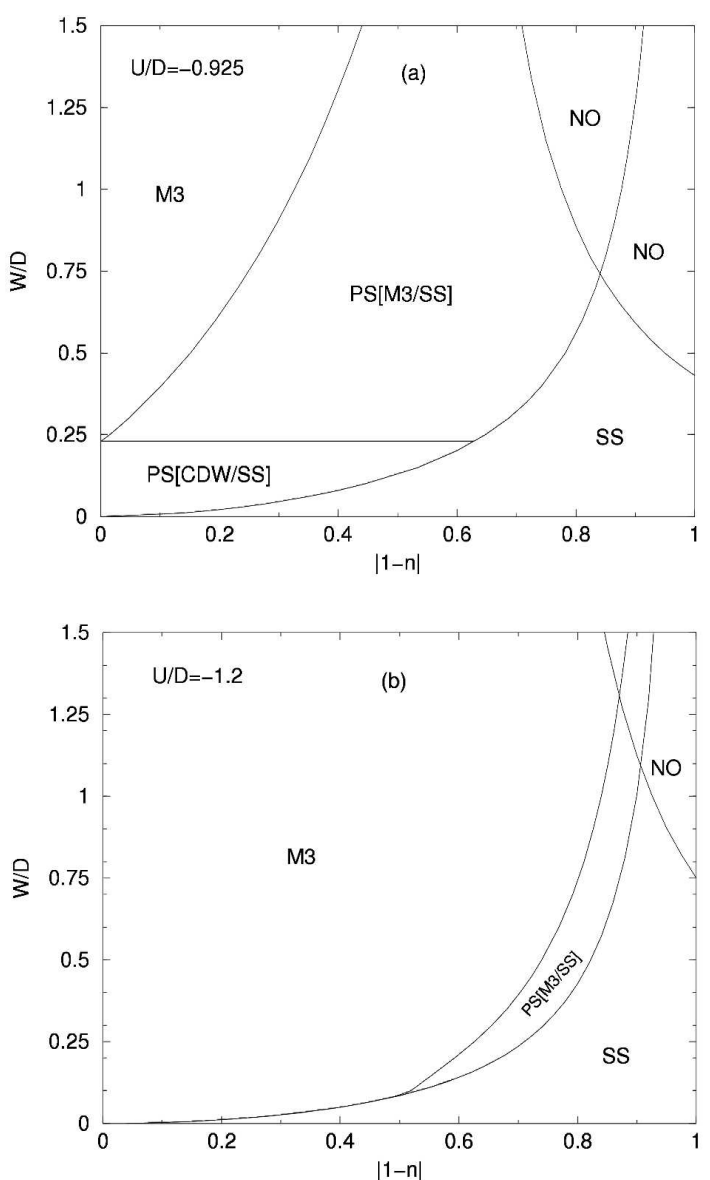

Fig. 2. Ground state phase diagram of model Eq. (9) calculated for the rectangular density of states for (a) $U=-0.925$ and (b) $U=-1.2$. For the $n=1$ the ground state is CDW phase, a smooth limit of both $\mathrm{M}(\mathrm{CDW}+\mathrm{SS}+\mathrm{SQ})$ and $\mathrm{PS}[\mathrm{CDW} / \mathrm{SS}]$ phases.

for $n \neq 1$. The states of the lowest energy are: SS, M3, PS[CDW/SS], and $\mathrm{PS}[\mathrm{M} 3 / \mathrm{SS}]$, and it will be so also for other values of parameters. In the inset there are the energy differences between the states: PS[CDW/SS], PS[M3/SS], and M3, difficult to discern in the main figure. We can see that the phase separated state $\mathrm{PS}[\mathrm{M} 3 / \mathrm{SS}]$ has the lowest energy of all the three (if exists). Its appearance is an unavoidable consequence of theorem 1 in the case when the phase M3 starts to win with PS[CDW/SS]. The phase diagrams are shown in Fig. 2a and b.

For not too large $|U|$ and $W$ the ground state is in PS[CDW/SS] or SS state, depending on $n$, in accordance with Ref. [11]. When we increase $W$ we reach a threshold, smallest for the half-filled band, above which the M3 phase is stabilized. Appearing of the M3 phase is connected with replacing PS[CDW/SS] 
by PS[M3/SS] phase, which is required by the Maxwell construction (see Eq. (5) and theorem 1). With increasing $|U|$ the threshold and the extent of PS[CDW/SS] phase are decreased and, for large enough $|U|$, the M3 phase is stabilized for all $W>0$ at $n=1$, as shown in Fig. 2b. The area of PS[CDW/SS] disappears at all, in favor of mixed phase.

In both Figs. 2a and b there is also a line "cutting off" the upper right-hand corner. This is the boundary for existence of bound pairs of extended $s$-wave symmetry, given by Eq. (2). In view of Randeria's notion [9], about necessity of such pairs for existence of superconductivity of this symmetry it can be used as an additional bound for the phase diagram [17]. Above these lines no superconductivity can survive so the NO phase enters diagram (to be sure of that we must consult again the free energy diagrams). Let us note that it is only an approximation, as we did not calculated extended $s$-wave superconductivity but only on-site, pure $s$. Preliminary results show nevertheless that the parameter of extended superconductivity $\Delta_{\gamma}$ is two orders of magnitude smaller than $\Delta_{0}$ along the boundary PS/SS state. Also $\eta$-pairing can have extended component, which will be stabilized by $W>0$, due to negative sign at the extended part of the pairing potential, unlike the case of extended $s$-wave superconductivity with $Q=0$. This fact will still enhance the main tendency outlined in this paper.

Inclusion of the normal Fock parameter into the calculations will bring about the increase in the band width, thus effectively pushing the system into weak-coupling limit. It can cause the increase in thresholds but should not change qualitatively the main results of this paper.

\section{Conclusions}

In the paper a phase diagram of the extended Hubbard model with attractive on-site and repulsive, nn, intersite interactions was analyzed in the Hartree-Fock approximation. A possibility of stabilizing the mixed phase in the model with short range, nn interactions only, was shown. Stabilization requires the existence of the three orderings: superconductivity, $\eta$-superconductivity, and charge density waves. The extent of the mixed phase increases with increasing $|U|$.

The other result of the paper comes from the solution of the Schrödinger equation for bound pairs of extended $s$-wave symmetry. The area of the normal phase is introduced into the phase diagram due to this criterion.

\section{Acknowledgments}

We acknowledge discussions with R. Micnas, S. Robaszkiewicz, T. Kostyrko, and support from the Foundation for Polish Science. This work was also supported by the State Committee for Scientific Research, project No. 1 P03B 08426. 


\section{References}

[1] R. Micnas, J. Ranniger, S. Robaszkiewicz, Rev. Mod. Phys. 62, 113 (1990).

[2] E.H. Lieb, F.Y. Wu, Phys. Rev. Lett. 20, 1445 (1968).

[3] Y. Nagaoka, Phys. Rev. 147, 392 (1966).

[4] A. Mielke, J. Phys. A 24, 3311 (1991).

[5] E.H. Lieb, Phys. Rev. Lett. 62, 1201 (1989); erratum: Phys. Rev. Lett. 62, 1927 (1989)

[6] T. Koma, H. Tasaki, Phys. Rev. Lett. 68, 3248 (1992).

[7] K. Kubo, K. Kishi, Phys. Rev. B 41, 4866 (1990).

[8] S.Q. Shen, Z.M. Qiu, G.S. Tian, Phys. Rev. Lett. 72, 1280 (1994).

[9] M. Randeria, J.-M. Duan, L.-Y. Shieh, Phys. Rev. Lett. 62, 981 (1989).

[10] P. Nozieres, S. Schmitt-Rink, J. Low Temp. Phys. 59, 195 (1985).

[11] S. Robaszkiewicz, G. Pawłowski, Acta Phys. Pol. A 90, 569 (1996).

[12] C.N. Yang, Phys. Rev. Lett. 63, 2144 (1989).

[13] K.A. Penson, M. Kolb, Phys. Rev. B 33, 1663 (1986).

[14] S. Robaszkiewicz, B. Bulka, Phys. Rev. B 59, 6430 (1999).

[15] A.S. Blaer, H.C. Ren, O. Tchernyshyov, Phys. Rev. B 55, 6035 (1997).

[16] S. Robaszkiewicz, R. Micnas, K.A. Chao, Phys. Rev. B 24, 4018 (1981).

[17] M. Bak, cond-mat/0406143. 